\title{
Reverse micellar system in protein recovery - a review of the latest developments
}

\begin{abstract}
Reversed micellar system (RMS) is an innovative technique used for the isolation, extraction and purification of proteins and enzymes. Studies have demonstrated that RMS is an efficient purification technology for extracting proteins and enzymes from natural plant materials or fermentation broth. Lately, reverse micelles have wider biological applications and the ease of scaling up and the possibility for the continuous process have made RMS a vital purification technique in various fields. In this study, an extensive review of RMS with the current application in biotechnology is examined. This review provides insights into the fundamental principles, key variables and parameters of RMS. In addition, a comparative study of RMS with other liquid-liquid extraction techniques is also included. The present review aims to provide a general overview of RMS by summarising the research works, since the introduction of the technology to current development.
\end{abstract}

Keyword: Reversed micellar system; Protein; Enzyme; Purification; Recovery; Genetic engineering 